\title{
Identification of Environmental Changes and Land Use in the Lagoon Ecosystems of Togo Southeastern
}

\author{
Ahlonko Koffi Bruce1 ${ }^{*}$, Selifa Sama², Kouami Kokou ${ }^{3}$ \\ ${ }^{1}$ Laboratoire de Botanique et d’Ecologie Végétale, Faculté des Sciences, Université de Lomé, Lomé, Togo \\ ${ }^{2}$ Département des sciences de la Terre, Faculté des Sciences, Université de Lomé, Lomé, Togo \\ ${ }^{3}$ Laboratoire de Botanique et d’Ecologie Végétale, Faculté des Sciences, Université de Lomé, Lomé, Togo \\ Email: ${ }^{*}$ michel.bruce@yahoo.fr
}

Received 1 October 2015; accepted 16 October 2015; published 21 October 2015

Copyright (C) 2015 by authors and OALib.

This work is licensed under the Creative Commons Attribution International License (CC BY).

http://creativecommons.org/licenses/by/4.0/

(c) (i) Open Access

\begin{abstract}
In the context of climate change and ecological disruptions, this study focuses on the diagnosis of the changes taking place in the microtidal environment of the Southeast lagoon ecosystems of Togo. To do this, a diachronic analysis of soil classification occupation of two Land sat satellite images (TM and ETM 1986 to 2011) was performed with ground verification. This analysis on two dates revealed the successive states to characterize the main environmental changes that have affected the lagoon ecosystems. The results revealed significant changes both natural and anthropogenic who achieved water resources and their buffer zones, plant cover and the frame of the study area. During 25 years, terrestrial vegetation around wetlands decreased by $26.28 \%$ and aquatic plants showed a surface increase of $\mathbf{1 3 . 2 \%}$. This study is a knowledge contribution to the lagoon ecosystems state in Togo through an ecological analysis which reveals some environmental changes in order to anticipate the loss of ecosystem goods and services of these wetlands.
\end{abstract}

\section{Keywords}

Lagoon Ecosystem, Environmental Change, Land Use, Togo

Subject Areas: Environmental Sciences, Hydrology

\section{Introduction}

Le paysage, espace géographique composé d’un ensemble d'écosystèmes en interaction, est dynamique [1]. Les écosystèmes sont des systèmes écologiques qui constituent les sièges de ces interactions. De ce point de vu, la compréhension de leur dynamique spatio-temporelle est indispensable en raison des multiples interactions 
environnementales avec les activités humaines. La structure spatiale des écosystèmes paysagers peut donc contribuer à éclairer les processus écologiques qui s’y déroulent [2].

Afin de réduire la vulnérabilité climatique et augmenter la résilience des populations riveraines, les milieux paraliques constituent aujourd'hui des écosystèmes de référence pour le suivi des changements climatiques [3] et des impacts anthropiques. Encore amplifiés par les modes et systèmes inappropriés d'exploitation des ressources disponibles, ces changements ont des répercussions directes sur l'occupation du sol et sur la configuration du paysage [4].

Au Togo, les écosystèmes lagunaires du Sud-Est du littoral font face à des pressions d'origine anthropique et naturelle qui tendent inexorablement à faire disparaître certaines zones humides.

De nombreuses études sur le système lagunaire [5] [6] ont mis en lumière les aspects biocénotiques [7] mais très peu sont parvenues à confronter la variabilité temporelle et spatiale aux paramètres biotiques et environnementaux.

Dans le même ordre d'idées, certains auteurs ont précédemment mis en exergue l'impact de l'accroissement démographique sur la dégradation des ressources naturelles [8]-[12]. C'est pourquoi, le suivi et la quantification de la dynamique de l'occupation du sol dans cette zone fortement peuplée et "vulnérable", s'avère nécessaire pour disposer des informations et susciter l'urgence d'une gestion intégrée et durable des écosystèmes lagunaires.

A partir de données diachroniques (cartes d'occupation du sol géoréférencées de 1986 et 2011) et de prospections de terrain, cette étude a pour but de montrer et de quantifier la dynamique dans le temps et dans l'espace de l'occupation du sol de cette zone du système lagunaire du Togo.

Il s'agit donc d'un diagnostic écologique afin d'anticiper sur les pertes des biens et services écosystémiques de ces zones humides.

\section{Materiel et Methode}

\subsection{Zone d'étude}

La zone étudiée est située au sud-est du Togo (Figure 1) drainée par un dense réseau hydrographique de type "éventail” (en couleur bleue sur la Figure 1). Deux principaux plans d'eau lagunaires y sont présents: le lac Togo et le lac Boko. Vers la frontière sud-est avec le Bénin, on observe le fleuve Mono, des mares et étangs permanents et/ou temporaires. Les cours d'eau ont un régime qui dépend de la quantité d'eau reçue dans l'année. Pendant les crues, ils débordent de leur lit et alimentent de véritables mares ou marécages.

\subsection{Démarche méthodologique}

La dynamique du paysage peut être décrite par des mutations dans le temps de sa composition et de sa structure spatiale. Dans le cas présent, le matériel utilisé dans cette étude comprend des images satellitales datant de 1986 à 2011 couvrant la zone d'étude. Elles proviennent des catalogues d'images d'archives issues des instruments TM et ETM+ correspondant respectivement aux satellites Landsat 5 et 7 . En plus des images satellitales, une carte topographique d'une échelle de 1: 200,000, élaboré à partir des photographies aériennes de 1979 par l'IGN France a été utilisée pour cette étude. La mission terrain a été menée avec pour but de reconnaître et de définir les éléments paysagers de la zone d'étude et d'effectuer des relevés de points GPS représentatifs de chaque classe d'occupation du sol précédemment définies. Les données ainsi obtenues devaient permettre d'aider à la compréhension des données satellitaires, puis de points de vérité terrain pour la validation de la classification la plus récente (janvier 2011). Environ 120 points ont été collectés le long de différents transects parcourus à pied et en voiture. Puis, un certain nombre de points jugés représentatifs ont permis de faire la correspondance avec la classe d'occupation du sol concernée et le nom du point tel qu'enregistré dans le GPS. Le caractère très hétérogène de l'occupation du sol de la zone d'étude a permis de choisir et travailler sur six classes (Table 1) pour mettre en œuvre la classification.

Chaque image d'une superficie de $185 \mathrm{~km} \times 185 \mathrm{~km}$, couvre le littoral du golfe de Guinée, du delta de la Volta (Ghana) et s'étend jusqu'au lac Nokoué (Bénin). Toutes les scènes sont projetées dans le système UTM/ WGS 84 zone $31 \mathrm{~N}$.

L’image Landsat TM acquise en 1986 (Figure 2) dispose d'une résolution spatiale de $30 \mathrm{~m}$. Par contre 


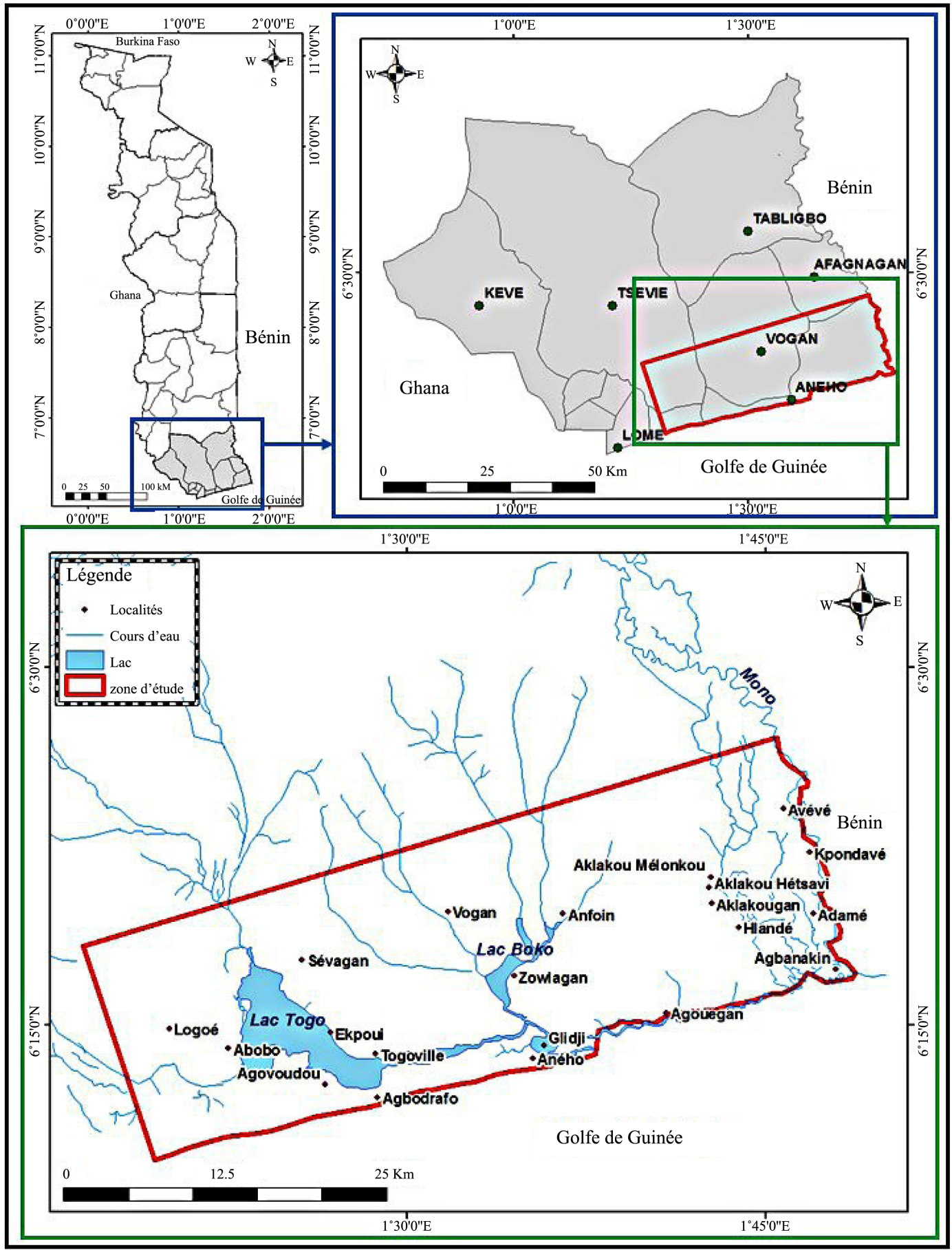

Figure 1. Localisation de la zone d'étude au sud-est du Togo.

l'image Landsat ETM+ de 2011 est constituée d'une bande panchromatique et de sept bandes multispectrales. Afin d'obtenir une meilleure classification d'image, plusieurs variables ont été utilisées à savoir: la signature spectrale, la transformée de l'image, les informations contextuelles, les images multitemporelles ou multisenseurs et les données auxiliaires. La séparabilité des parcelles d'entrainement a été effectuée avec la méthode de la transformée de la divergence. La meilleure séparabilité des parcelles d'entrainement par la méthode de la transformée de la divergence est de 2000. A cet effet, une séparabilité acceptable a été observée car la valeur moyenne de séparabilité de l’image de janvier 1986 est de 1990 et celle de janvier 2011 de 1999. 


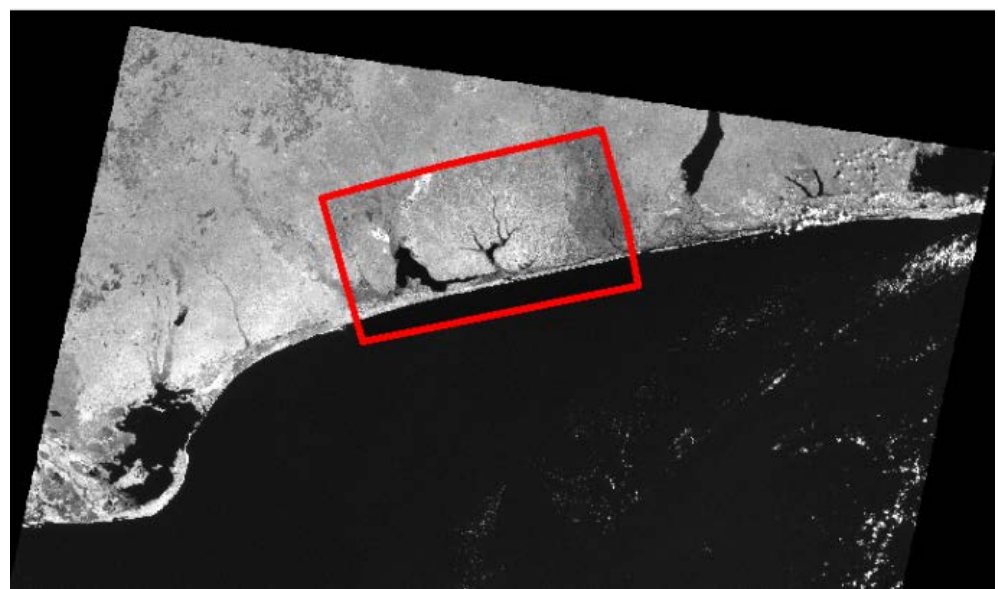

Figure 2. Image Landsat de 1986 montrant la zone d’intérêt (en encadré).

Table 1. Identification et définition des classes d’occupation du sol présentes dans la zone d'étude.

\begin{tabular}{|c|c|}
\hline Classe & Définition \\
\hline $\begin{array}{l}\text { Végétation terrestre } \\
\text { (naturelle/cultivée) }\end{array}$ & $\begin{array}{l}\text { Surfaces cultivées. d’étendues variables situées à proximité des eaux lagunaires et des habitations. } \\
\text { Distinction visible entre la végétation naturelle et la végétation cultivée. } \\
\text { Ancienne parcelle cultivée laissée à l'abandon avec un défrichement progressif. }\end{array}$ \\
\hline Végétation aquatique & $\begin{array}{l}\text { plantes aquatiques formant de denses tapis généralement monospécifiques. } \\
\text { Elles sont adaptées aux conditions d'engorgement et de salinité dont l'intensité diminue à } \\
\text { mesure que l'on s'éloigne des eaux libres ou à la faveur d'une arrivée d'eau douce. }\end{array}$ \\
\hline Sol nu & $\begin{array}{l}\text { Classe qui comprend les terrains de football. coure d'école...Il s’agit de sol nu. } \\
\text { rouge. qui se recouvre d'herbe dans la saison des pluies. } \\
\text { Confusion avec les parcelles de cultures à nu. }\end{array}$ \\
\hline Sol humide & $\begin{array}{l}\text { Sol se caractérisant par la présence d’eau disponible. permanente ou temporaire. } \\
\text { en surface ou à faible profondeur dans le sol. }\end{array}$ \\
\hline Eau continentale & $\begin{array}{l}\text { Il s'agit des plans d'eau lagunaire pouvant communiquer soit. } \\
\text { de manière continue avec des valeurs hydrauliquement faibles. soit de manière discontinue. }\end{array}$ \\
\hline Bâti & Comprend les habitations et infrastructures. \\
\hline
\end{tabular}

Contrairement à l'image Landsat ETM+, il faut rappeler que l'image Landsat TM de 1986 ne dispose pas de bande panchromatique, par conséquent les bandes spectrales de la longueur d'onde du bleu $(0.45-0.52 \mu \mathrm{m})$ présentant une importante diffusion et la longueur d’onde du thermique (10.4 - $12.5 \mu \mathrm{m})$ représentant l'émission terrestre n’ont pas été retenues pour les analyses. Les fonctions radiométriques améliorent l'image en utilisant les valeurs de chaque pixel dans chaque bande.

Par ailleurs, l'algorithme du Maximum de vraisemblance a été appliqué. Cette méthode repose sur les caractéristiques statistiques des échantillons représentatifs des classes précédemment identifiées sur l’image. L'algorithme affecte chaque pixel de l'image à la classe d'occupation du sol à laquelle il a la plus forte probabilité d'appartenir [13]. Au total, pour chaque classification, les classes d'occupation du sol précédemment définies ont pu être discriminées.

Enfin, la précision des classifications obtenues a été évaluée à travers l'utilisation d'une matrice de confusion. Pour ce faire, elles ont été confrontées à des relevées terrains extraits d’images Google Earth.

Deux indices de validation de classification sont employés:

- La précision globale: caractérise la proportion de pixels bien classés, calculé en pourcentage ;

- Une estimation du coefficient Kappa a été réalisée pour caractériser le rapport entre les pixels bien classés et le total des pixels.

Le calcul de l'évolution des unités d’occupation du sol à différentes périodes [14] a été utilisé. À partir des images classifiées, la dynamique des différentes classes d’occupation du sol a été estimée.

La déduction des changements survenus au sein des différentes unités d’occupation du sol a permis de les 
qualifier, de les quantifier et de les localiser à l'aide de la nomenclature précédemment définie (Table 1).

\section{Resultats}

\subsection{Mutations de l'occupation du sol entre 1986 et 2011}

Les classifications supervisées des images de 1986 et 2011 ont permis de distinguer six classes d'entités qui correspondent à des types d'occupation du sol (Table 2): Sol humide, végétation aquatique, végétation terrestre, sol nu, bâti et eau continentale.

Le Table 2 donne les pourcentages des mutations opérées entre les différentes classes d'occupation du sol entre 1986 et 2011 (Figure 3 et Figure 4) dans la zone d'étude. Les résultats montrent que le taux de végétation aquatique est passé de $1.90 \%$ en 1986 à $15.09 \%$ en 2011. Le taux de l'eau continentale qui était de $19.74 \%$ en 1986 est tombé à 7.42\% en 2011. Quant au bâti, son occupation du paysage est passée de $13.77 \%$ en 1986 à $25.29 \%$ en 2011. En règle, les taux d'occupation des classes sol humide, végétation aquatique, sol nu et bâti ont augmenté entre 1986 et 2011. Cette augmentation s'est principalement opérée au détriment de l'eau continentale.

Sachant qu'une étude portant sur l'occupation des terres peut être validée si l'indice de Kappa est compris entre $50 \%$ et $75 \%$ [15], alors il est cohérent de déduire que les indices de Kappa des images de janvier 1986 et de janvier 2011 respectivement de $72.86 \%$ et de $69.70 \%$ sont corrects.

\subsection{Analyse de la dynamique spatiale entre 1986 et 2011}

Le Table 2 récapitule les différentes transformations des unités d’occupation du sol calculé dans chacune des six classes en 1986 et en 2011. L'analyse des résultats issus des Figure 3 et Figure 4 corroborée par la réalité terrain révèlent trois grands processus environnementaux qui se sont déroulés dans le paysage étudié en 25 ans. Autrement, la matrice des changements résultant du croisement entre la classification de 1986 et celle de 2011 permet d'identifier quelques dynamiques:

- Premièrement, l'envahissement des plans d'eau lagunaires par les végétaux aquatiques $(+13.2 \%)$ se traduisant par la régression surfacique de l'eau continentale (-12.32\%). Les observations de terrain (Photo 1 et Photo 2) révèlent la prolifération de végétaux à croissance rapide.

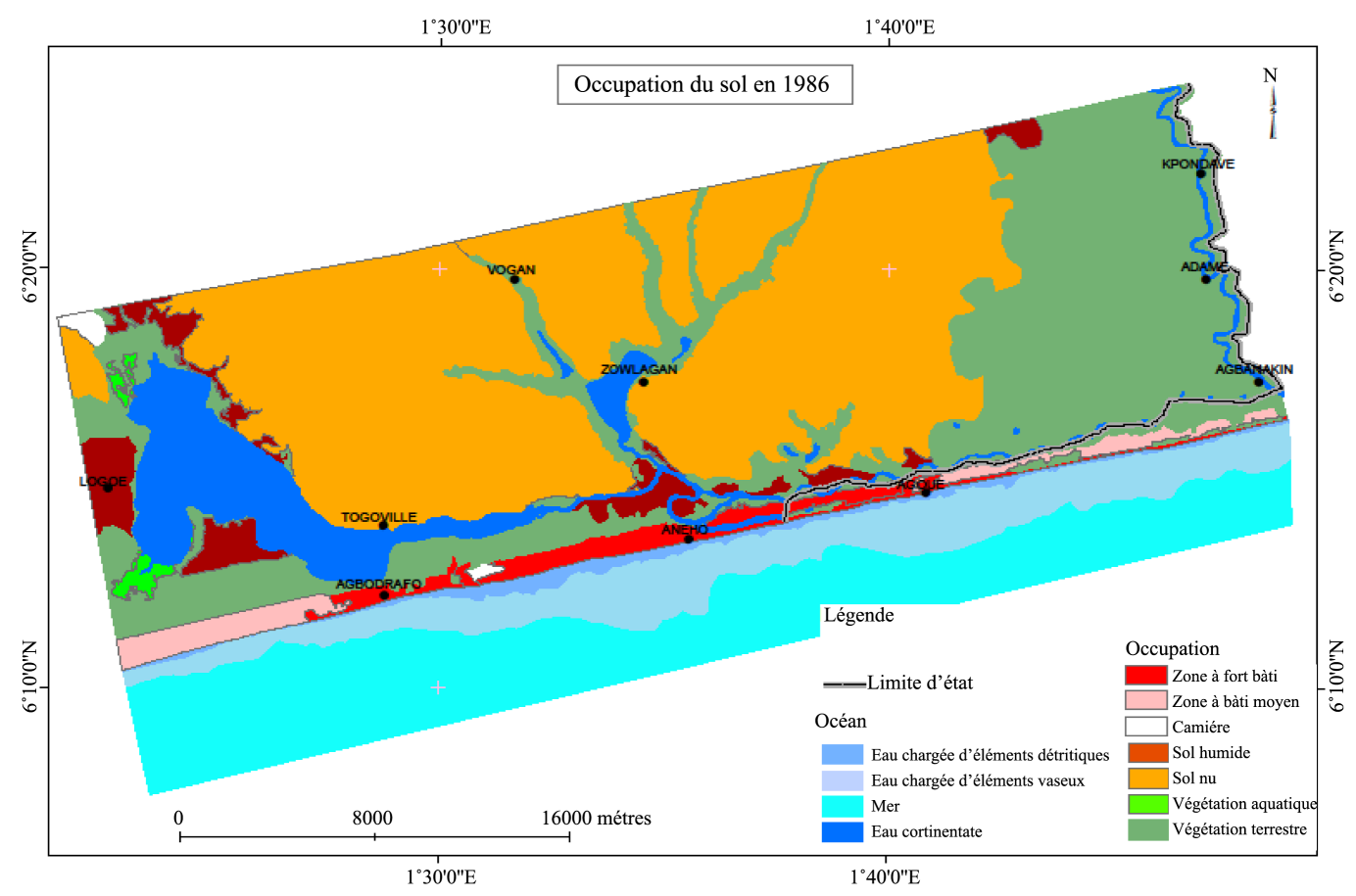

Figure 3. Etat d’occupation du sol des écosystèmes lagunaires au sud-est du Togo en 1986. 


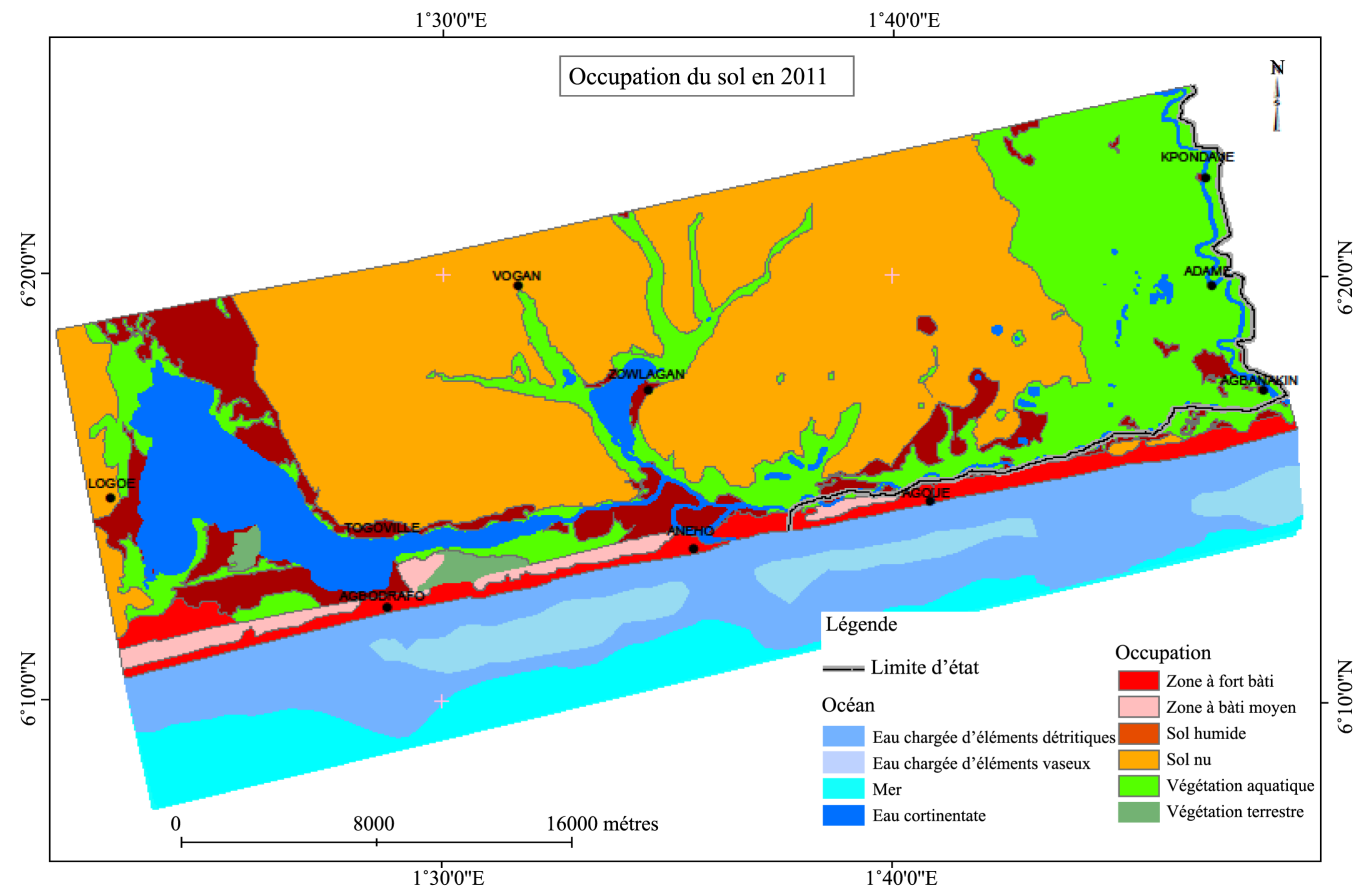

Figure 4. Etat d’occupation du sol des écosystèmes lagunaires au sud-est du Togo en 2011.

Table 2. Occupation du sol obtenue à partir des classifications supervisées des images de 1986 et 2011.

\begin{tabular}{cccccccccc}
\hline Type d'occupation & \multicolumn{2}{c}{ Etat en 1986 } & \multicolumn{2}{c}{ Etat en $\mathbf{2 0 1 1}$} & \multicolumn{2}{c}{$\begin{array}{c}\text { Progression } \\
\text { (1986-2011) }\end{array}$} & \multicolumn{2}{c}{ Régression (1986-2011) } \\
\hline Unité d'occupation du sol & en ha & en \% & en ha & en \% & en ha & en \% & en ha & en \% \\
\hline Sol humide & 5588 & 6.13 & 8461.4 & 9.28 & 2873.5 & 3.15 & - & - \\
Végétation aquatique & 1735.7 & 1.90 & 13754.6 & 15.09 & 12019 & 13.2 & - & - \\
Végétation terrestre & 24502.3 & 26.88 & 546.7 & 0.60 & - & - & -23955.6 & -26.28 \\
Sol nu & 28790.8 & 31.58 & 38578.7 & 42.32 & 9787.9 & 10.74 & - & - \\
Bâti & 12551.4 & 13.77 & 23061.5 & 25.29 & 10510.1 & 11.52 & -1234.7 & -12.32 \\
Eau continentale & 17998.7 & 19.74 & 6764 & 7.42 & - & - & -11234 \\
TOTAL & $\mathbf{9 1 1 6 6 . 9}$ & 100.00 & $\mathbf{9 1 1 6 6 . 9}$ & 100.00 & - & - & - & - \\
\hline
\end{tabular}

Aussi, l'évolution de la pluviométrie au Togo se traduit par une tendance générale à la baisse de la pluviométrie et à l'irrégularité des pluies marquée par une élévation des températures. Cette variabilité climatique entrainerait par ailleurs un accroissement de l'évapotranspiration affectant directement les eaux de surface.

- Deuxièmement, le constat de la dégradation de la végétation terrestre (-26.28\%) autour des plans d'eau. Sur 25 ans, on dénote une conversion des terres d'environ 24000 hectares s'expliquant par une importante mise en culture de la végétation naturelle riveraine et par le surpâturage (Photo 3 et Photo 4).

L'analyse diachronique des images photo-interprétées et l'analyse spatiale ont permis d'estimer l'augmentation surfacique des sols nus à 9787.9 hectares de 1986 à 2011. Les sols nus se caractérisent in situ par des terrains entièrement inoccupés et constitués essentiellement de sable, d'argile, de limons mais aussi de mangroves et marais. Par ailleurs, la classe "sol humide" marquée par une faible variation surfacique $(+3.15)$ entre 1986 et 2011 se caractérise par le stockage de l'eau dans le sol afin de favoriser une diminution de l'intensité des crues et des dommages causés par les inondations (cas de la vallée inondable du fleuve Mono). L'eau accumulée pendant les périodes pluvieuses ou lors d'évènements météorologiques exceptionnels pourrait 


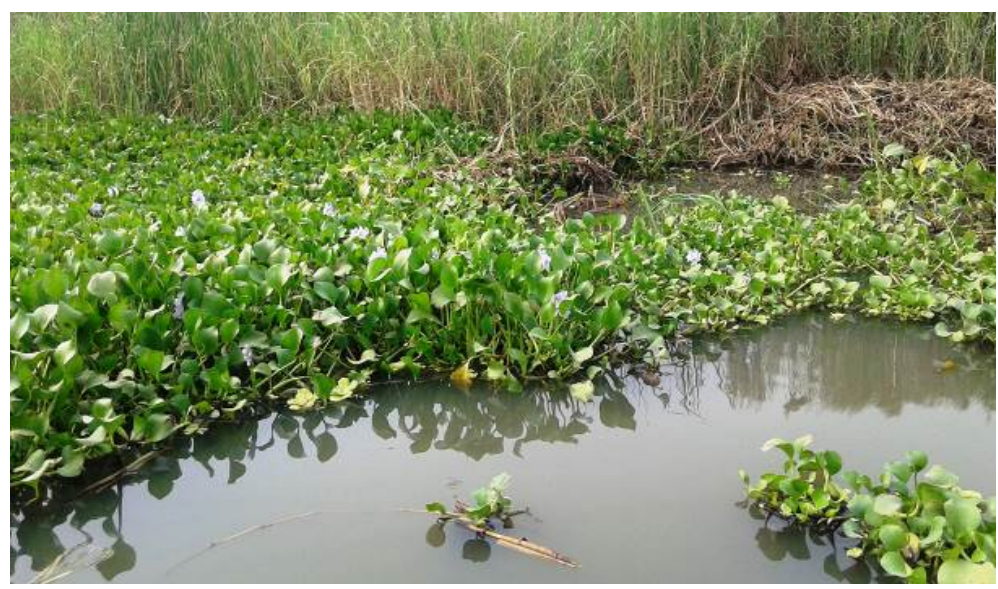

Photo 1. Prolifération de végetaux aquatiques (Ecchornia crassipes) sur le lac Togo près du village Togoville.

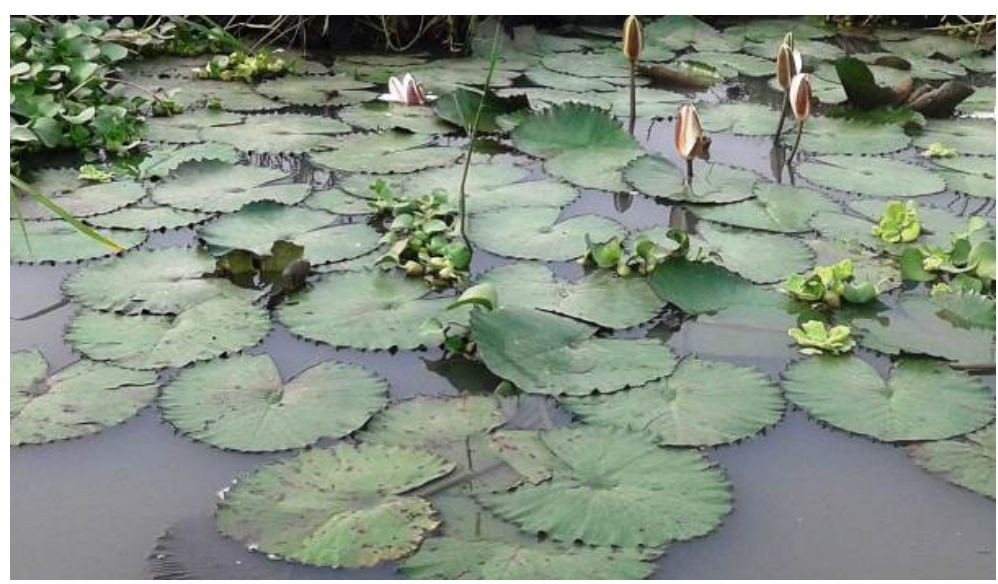

Photo 2. Prolifération de végetaux aquatiques (Pistia stratoïtes et Ecchornia crassipes) sur le lac Boko près de Zowlagan.

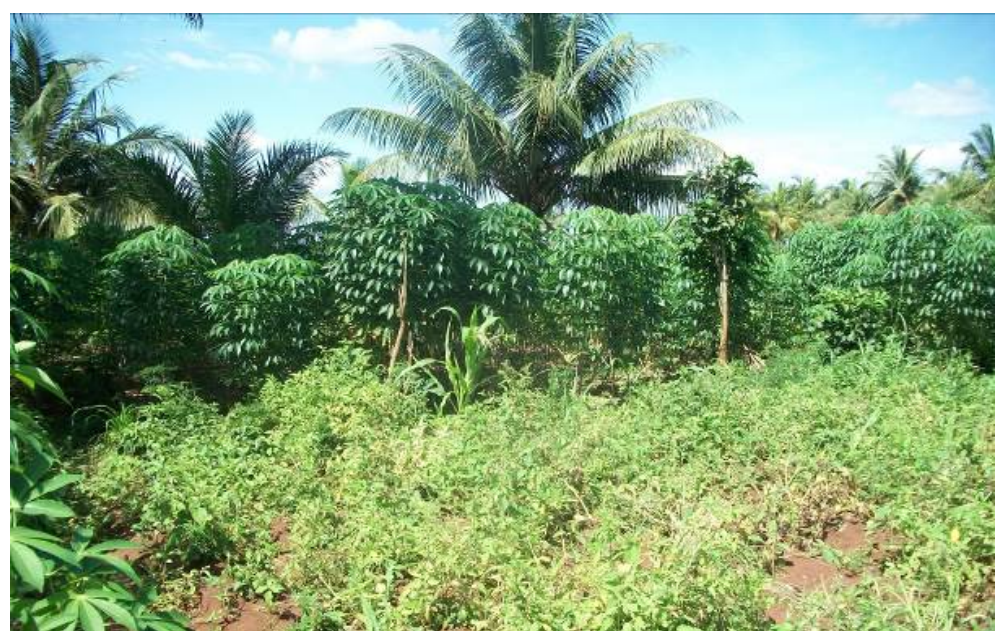

Photo 3. Cultures vivrières sur les berges du lac Togo près de Togoville.

alimenter progressivement les nappes phréatiques et les cours d'eau pendant les périodes sèches comme celles de janvier 1986 et janvier 2011. 


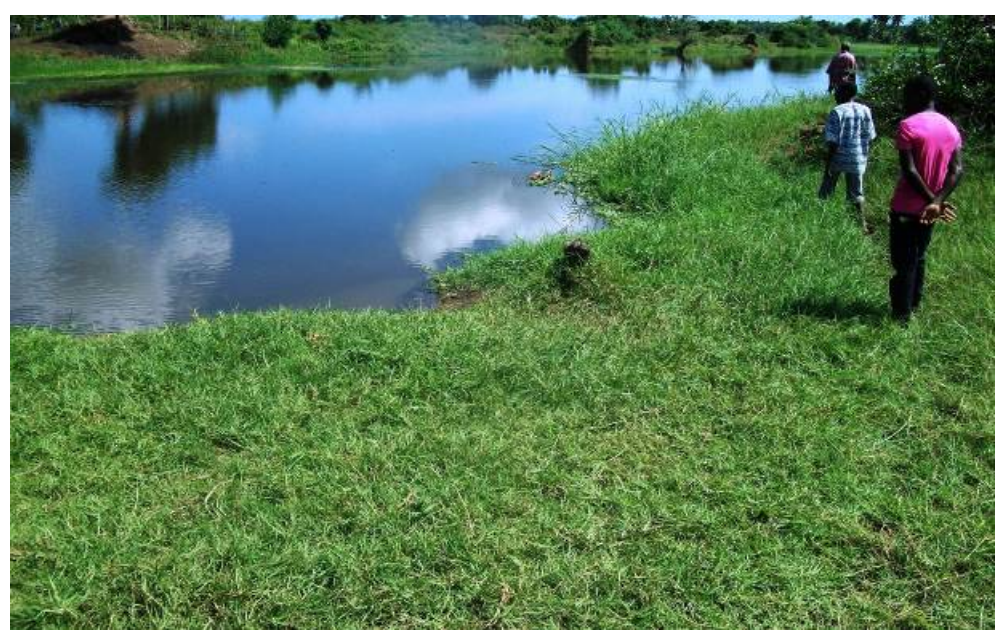

Photo 4. Berges dégradées par surpâturage au niveau du lac Boko près de Zowlagan.

\section{Discussion}

Dans un contexte de pressions croissantes exercées sur les ressources naturelles, l'objectif de ce travail était de mettre en place une analyse diachronique de l'occupation du sol entre 1986 et 2011 dans les écosystèmes lagunaires du sud-est du Togo.

La méthode basée sur les traitements post-classification a permis d'analyser les modalités de transition entre les différentes dates à l'aide de capteurs différents (ETM + et TM). Les images Landsat ont permis de mettre en place les cartographies de l'occupation du sol. L'utilisation de 4 à 6 classes d'occupation du sol est bien souvent suffisante pour mettre en place une analyse cartographique sur ce genre de paysage [16].

Dans le cadre de cette étude, la forte nébulosité pendant la saison pluvieuse a été une contrainte fondamentale dans l'utilisation et le choix des images acquises pendant la saison sèche. Par conséquent, la discrimination entre certaines parcelles de cultures des classes "végétation terrestre" et "sol nu" a été rendu difficile.

La prolifération des végétaux aquatiques $(+13.2 \%)$ s'expliquerait par un apport excessif en nutriments (nitrates et phosphates agricoles, eaux usées, etc.). Ceci proviendrait de toute évidence de l'utilisation non rationnelle des engrais agricoles (riche en azote et phosphore) qui sont à leur tour lessivés vers les eaux lagunaires dont les berges sont déjà fortement dégradées (villages Togoville, zowlagan, Ekpoui). Cette perturbation écologique constitue un indicateur de l'eutrophisation des eaux lagunaires tel que confirmé par les travaux de [17] et [18].

La végétation naturelle agressée de toutes parts autour des eaux lagunaires en période sèche devrait s'expliquer avec la forte dépendance hydrique des activités agricoles. Cette situation serait due à une pratique non durable du système agraire, elle-même exacerbée dans le cas de notre zone d'étude, par une pression foncière (+11.52) et démographique de plus en plus croissante.

D’environ 24,500 hectares en 1986, la végétation naturelle est passée à 547 hectares en 2011. Tout porte à croire que cette classe devrait encore diminuer dans les mois et années à venir, si la tendance se poursuit. Aussi, la réalité terrain a permis de constater qu'on assiste dans certains cas à la conversion des zones de végétation naturelle en zone de cultures (villages Ekpoui, Togoville, Abobo) ou à la conversion des zones de cultures en zone d’habitation (Togoville, Adamé, Kpondavé, Aklakougan, Zowlagan et Glidji).

La pression anthropique est en grande partie responsable du changement d'occupation de l'espace. Les contraintes climatiques combinées aux différentes activités humaines notamment les pratiques culturales incontrôlées favorisent la dégradation des milieux lagunaires du sud-est du Togo.

L'augmentation continue du bâti de près de 11,000 hectares en 25 ans traduit un accroissement des besoins en terre et par conséquent impliquerait une corrélation avec la pression démographique.

\section{Conclusion}

Cette étude a révélée qu'en 25 ans, la végétation terrestre autour des zones humides a régressé de $26.28 \%$ et les 
végétaux aquatiques ont connu une progression surfacique de 13.2\% sur les plans d'eau. L'analyse des images Landsat de 1986 et 2011 a permis de suivre l'évolution de quelques écosystèmes lagunaires du Sud Est du Togo. Outre les variabilités climatiques et leurs incidences, il ressort de cette étude que les pressions anthropiques et la poussée démographique explique essentiellement les changements surfaciques observés sur 25 ans. De ce point de vue, les résultats de cette étude traduisent que la pression foncière agricole, la pression démographique, l'urbanisation et l'eutrophisation de ces milieux participent activement à la régression et la dégradation des lagunes du sud-est du Togo. Les divers constats visuels et autres changements environnementaux observés dans le cadre de cette étude démontrent l'urgence d'une gestion rationnelle et participative des eaux du système lagunaire du Togo.

Enfin, ces constats viennent conforter la nécessité d'un suivi environnemental continu à deux niveaux: (i) celui de l'environnement immédiat des plans d'eau lagunaire correspondant actuellement à leurs zones d'influence directe; (ii) et, celui du statut trophique des eaux lagunaires.

\section{References}

[1] Bogaert, J. et Mahamane, A. (2005) Ecologie du paysage: cibler la configuration et l'échelle spatiale. Annales des Sciences Agronomiques du Bénin, 1, 39-68.

[2] Fortin, M.J. (2002) Analyse spatiale en écologie: Problèmes statistiques et de mise à l’échelle au niveau du paysage. Ecoscience 9: iii-v.

[3] Castaings, J. (2008) Etat de l'art des connaissances du phénomène de comblement des milieux lagunaires, Rapport de phase $1,115 \mathrm{p}$.

[4] Bamba, I., Ama, A., Neuba, D., Koffi. K., Traoré, D., Visser, M., Sinsin, B., Lejoly, J. et Bogaert, J. (2008) Influence des actions anthropiques sur la dynamique spatio-temporelle de l'occupation du sol dans la province du Bas-Congo (R.D. Congo). Sciences \& Nature, 5, 49-68.

[5] Laibi, R.A., Gardel, A. et Anthony, E.J. (2012) Apport des séries d'images LANDSAT dans l'étude de la dynamique spatio-temporelle de l'embouchure de l'estuaire des fleuves Mono et Couffo au Bénin, avant et après la construction du barrage de Nangbéto sur le Mono. Revue Télédétection, 10, 179-188.

[6] Badahoui, A., Fiogbe, E.D. et Boko, M. (2010) Les causes de la dégradation du lac Ahémé et ses chenaux. International Journal of Biological and Chemical Science, $16 \mathrm{p}$.

[7] Millet, B. (1986) Hydrologie et hydrochimie d'un milieu lagunaire tropical: Le lac Togo. Etudes et Thèses, ORSTOM, Paris, Th. 3e cycle, 228 p.

[8] Maiguet, M. (1991) Desertification: Natural Background and Human Mismanagement. Springer-Verlag, Berlin, 306p. http://dx.doi.org/10.1007/978-3-642-97253-9

[9] Kokou, K. (1998) Les mosaïques forestières au sud du Togo: biodiversité, dynamique et activités humaines. Thèse de Doctorat. Université de Montpellier II, Montpellier, France. 1998, 139 p. + annexes

[10] Mathieu, P. (2001) Accroissement démographique et gestion de l'environnement en Afrique Sub-Saharienne: développement durable ou catastrophes inéluctables. Population et développement, 375-412.

[11] Ariori, S.L. (2004) Déforestation en Afrique de l'ouest: Mise au point et implications pour la désertification. Mémoire. Université de Liège/Faculté Universitaire des Sciences Agronomiques de Gembloux, Belgique, 42 p.

[12] Ozer, P. (2004) Introduction aux risques naturels. Note de cours, Département des Sciences et Gestion de l’Environnement, Université de Liège, Arlon, Belgique, b, 56 p.

[13] Robin, M. (2002) Télédétection, Des satellites au SIG. Une analyse complète du processus de création d'un type essentiel d'information géographique. Nathan Université. 318 p.

[14] Akognongbe, A., Abdoulaye, D., Vissin, E.W. et Boko, M. (2014) Dynamique de l'occupation du sol dans le bassin versant de l’Ouémé à l'exutoire de Bétérou (Bénin), Afrique SCIENCE 10(2) (2014) 228-24, 15 p.

[15] Pontius, R.G. (2000) Quantification error versus location error in comparison of categorical maps. Photogrammetric Engineering and Remote Sensing, 66, 1011-1016.

[16] Leroux, L. (2012) Analyse diachronique de la dynamique paysagère sur le bassin supérieur de l’Ouémé (Bénin) à partir de l'imagerie Landsat et MODIS-Cas d'étude du communal de Djougou, Hydrosciences Montpellier, ANR ESCAPE, $62 \mathrm{p}$.

[17] Dongo, K.R., Niamke, B.F. et Adje, A.F. (2013) Impacts des effluents liquides industriels sur l'environnement urbain d'Abidjan-Côte d'Ivoire. African Journal Online (AJOL), 17 p.

[18] Amoussou, E. (2010) Variabilité pluviométrique et dynamique hydro-sédimentaire du bassin-versant du complexe fluvio-lagunaire Mono-Ahémé-Couffo (Afrique de l’Ouest). Thèse de doctorat Université de Bourgogne, Bourgogne, France, $313 \mathrm{p}+$ annexes. 